\title{
Prevenção da mortalidade materna: desafio para o enfermeiro
}

\author{
Maternal mortality prevention: a challenge for nurses \\ Prevención de la mortalidad materna: un desafío para la enfermera
}

\begin{abstract}
Patrícia Santos Barbastefano', Octavio Muniz da Costa Vargens
'Universidade Estadual do Rio de Janeiro. Faculdade de Enfermagem. Programa de Pós-Graduação. Núcleo de Estudos e Pesquisas Enfermagem, Mulher, Saúde e Sociedade. Rio de Janeiro, RI
\end{abstract}

Submissão: 3 1/08/2008

Aprovação: 03/03/2009

\section{RESUMO}

Neste artigo analisamos os principais aspectos sobre a mortalidade materna, tendo como objetivo a promoção de ações preventivas para a morte materna evitável. Verifica-se Que entidades como a ADVOCACY têm significativa participação nos projetos visando redução das taxas de mortalidade materna e o ajustamento de condutas de proteção aos direitos da mulher. Observa-se ainda Que a SES, através das Resoluções no 1.052/95 e no 1.642/2001, expressa a política estadual para redução do problema. Conclui-se Que há indícios de vontade política nas propostas e projetos para redução das taxas de mortalidade materna evitável, porém não há cobrança da sua efetividade. Profissionais da saúde como o enfermeiro, precisam reunir esforços, conscientização e sensibilização em suas ações preventivas.

Descritores: Cuidados de enfermagem; Enfermagem obstétrica; Mortalidade materna; Saúde da mulher.

ABSTRACT

In this article theprincipal aspects about maternal mortality are analyzed, with the objective the promotion of preventive actions for the avoidable maternal death. It is verified that entities as ADVOCACY have significant participation in the projects seeking for reduction of maternal mortality rates and the adjustment of procedures for protecting women's rights. It is also observed that the State Health Secretary, through the Resolutions 1.052/95 and 1.642/200 I express the state policies for reducing the problem. It is concluded that there are indications of political will about the proposals and projects for reducing the avoidable maternal mortality rates. However, there are no requests on its effectiveness. Health professionals as nurses need to gather efforts, and also an attitude of understanding and sensitiveness in their preventive actions.

Descriptors: Nursing care; Obstetric nursing; Maternal mortality; Women’s health.

\section{RESUMEN}

En este artículo se analizan los aspectos principales sobre mortalidad maternal Que tiene como objetivo la promoción de acciones preventivas para la muerte materna evitable. Se verifica Que las entidades como la ADVOCACY tienen participación significante en los proyectos Que buscan la reducción de la tasa de mortalidad materna y el ajuste de procedimientos de protección de los derechos de la mujer. También se observa que la Secretaria Estatal de Salud, a través de las Resoluciones n 1.052/95 y nº l.642/2001, expresa las políticas estatales para la reducción del problema. Se concluye que hay indicaciones de voluntad política sobre las propuestas y proyectos para reducir las tasas de mortalidad materna evitables. Sin embargo no hay demandas sobre su efectividad. Los profesionales de salud como enfermeras necesitan recoger los esfuerzos, y también una actitud de comprensión y sensibilidad en sus acciones preventivas.

Descriptores: Atención de enfermería; Enfermería obstétrica; Mortalidad materna; Salud de la mujer. 


\section{INTRODUÇÃO}

O ser humano, social por excelência, está ligado à necessidade gregária, onde a família, considerada como célula mater das sociedades, cumpre seu papel designado pelo Criador (crescei e multiplicai-vos). Isso garante ao casal, não só a tarefa da perpetuação da espécie humana, como o direito ao bom desempenho dessa função, incluindo reconhecimento, dignidade, amparo ao crescimento e desenvolvimento saudável da prole, baseado no apoio à gestação e parto dentro de parâmetros preventivos. No âmbito da saúde materno-infantil, esta prevenção constitui desafio para o enfermeiro Que, prescindindo de vontade política dos governantes, é um dos profissionais da saúde atuantes nos programas voltados para a redução dos índices de mortalidade materna.

De acordo com o Relatório do Comitê Estadual de Prevenção e Controle da Morte Materna e Perinatal, da SES/RJ, morte materna é a morte de uma mulher durante a gestação ou dentro de um período de 42 dias após o término da gestação, independente da duração ou da localização da gravidez, devida a Qualeuer causa relacionada com ou agravada pela gravidez ou por medidas em relação a ela, porém não devidas a causas acidentais ou incidentais $(\mathrm{TMM}=\text { Taxa de Mortalidade Materna })^{(1,2)}$.

A relação entre mortalidade materna e direitos humanos aponta violação dos direitos humanos das mulheres, face a omissões e silêncio do Estado ${ }^{(3)}$. Desde 2002 a Advocacy, organização não governamental, fundada em 2001 , ,ue tem como objetivo fomentar o uso estratégico do direito como instrumento de justiça social, vem acompanhando as iniciativas da Secretaria de Estado de Saúde e do Ministério Público do Estado, para a implementação de políticas de saúde relevantes para a redução da mortalidade materna e neonatal no Estado, iniciadas no ano $2000^{(4)}$.

Deste modo, definiu-se como objetivo deste estudo analisar a Questão da mortalidade materna em termos de reunião de esforços oficiais e dos profissionais da saúde envolvidos, destacando-se o papel do enfermeiro obstetra e seus desafios de contribuição à Qualidade de vida e saúde da mulher em idade fértil.

\section{METODOLOGIA}

A metodologia de elaboração deste artigo seguiu os pressupostos da revisão de literatura ${ }^{(5)}$, cujos procedimentos metodológicos incluíram levantamento bibliográfico, seleção de textos, fichamentos, estruturação preliminar, estruturação lógica do estudo, revisão, construção da conclusão e da introdução.

\section{RESULTADOS E DISCUSSÃO}

\section{O Problema da Morte Materna na Perspectiva das Políticas Públicas no Brasil}

Em 2001 ocorreram 174 mortes maternas: destas, 173 de residentes no próprio Estado do Rio de laneiro e I de mulher residente em outro Estado. A Taxa de Mortalidade Materna (TMM) para o ano foi de 71,5 óbitos para cada 100.000 nascidos vivos. Continua sendo uma taxa considerada alta e chega a "ser mais que dez vezes maior do Que os valores em países desenvolvidos"(1).

Entre os princípios e diretrizes do PHPN, conforme já assinalado, estão: o direito ao acesso e atendimento digno e de Qualidade no decorrer da gestação, parto e puerpério; o direito ao acompanhamento pré-natal adequado; o direito de saber e ter assegurado o acesso à maternidade em Que será atendida no momento do parto; o direito à assistência ao parto e ao puerpério, e Que esta assistência seja realizada de forma humanizada e segura; o direito de todo recém-nascido ter uma assistência neonatal adequada; e a responsabilização das autoridades sanitárias dos âmbitos federal, estadual e municipal na promoção e garantia dos direitos enunciados ${ }^{(4)}$.

Este programa vem sendo descrito por gestores de saúde, profissionais de saúde e feministas, como um programa estratégico Que pode influenciar na redução das taxas de mortalidade materna. Apesar de ser uma política pública importante, o PHPN não tem sido eficaz para a redução da mortalidade materna, em parte porQue não está focalizado na eficácia e segurança dos procedimentos no parto. Além disso, a sua adesão tem sido lenta pelos municípios, segundo dados de $2002^{(1)}$.

Tais iniciativas contemplaram dois momentos distintos: o da assinatura dos Termos de Ajustamento de Conduta pelos Municípios, Ministério Público Estadual e Secretaria de Estado de Saúde, e a adesão dos Municípios ao PHPN, através da assinatura do Termo de Adesão junto à Secretaria de Estado de Saúde. A alimentação do sistema de dados do Ministério da Saúde (SISPRENATAL, software desenvolvido pelo Datasus, com a finalidade de permitir o acompanhamento adequado das gestantes inseridas no Programa de pré-natal), é um dos caminhos para o monitoramento do efetivo cumprimento dos compromissos assumidos pelos municípios, já Que a adesão ao programa significa alocação de recursos a este.

Em julho de 2003, foram elaborados requerimentos pela Advocacy apresentados aos representantes do Ministério Público, em conjunto com a Rede Nacional Feminista de Saúde, Direitos Sexuais e Direitos Reprodutivos - Regional Rio, visando novos Termos de Ajustamento de Conduta relacionados à situação da mortalidade materna, e aos serviços de planejamento familiar no Estado. Infelizmente, até o presente, ainda são registrados casos de mortes maternas evitáveis ${ }^{(2,3)}$.

A Secretaria de Estado de Saúde do Rio de Janeiro (SES-RI), através da ação conjunta do PAISMCA e do Departamento de Dados Vitais (DDV) vem, desde 1995, em integração com as Secretarias Municipais de Saúde, implementando a vigilância da morte materna através da busca ativa de óbitos, melhorando as informações através do trabalho do Comitê Estadual de Prevenção e Controle da Morte Materna e Perinatal (CEPCMMP), e desenvolvendo ações para Qualificar a atenção à gestação, parto e puerpério. A mortalidade materna é, há alguns anos, a $9^{a}$ causa de morte de mulheres em idade fértil, no Estado do Rio de Janeiro ${ }^{(1)}$.

A sub-declaração da causa de morte materna não é um problema exclusivo do Estado do Rio ou dos países em desenvolvimento. A Organização Mundial de Saúde (OMS), preconiza um fator de correção de 1,5 para os óbitos maternos ocorridos nos países desenvolvidos, e com sistema de registro de eventos vitais considerados de boa Qualidade.

O trabalho conjunto da SES-RI, através do Programa de Assistência Integral a Saúde da Mulher, Criança e Adolescente e do Departamento de Dados Vitais, e das Secretarias Municipais de Saúde, tem estimulado a identificação e a busca ativa dos óbitos. 
Somente o cumprimento das Resoluções SES no 1.052/1995 e SES n ${ }^{\circ} 1.642 / 2001$ poderá estabelecer uma Taxa de Mortalidade Materna mais próxima do real. Essas Resoluções, respectivamente, dispõem sobre a obrigatoriedade de informar, no prazo máximo de $24 \mathrm{~h}$, a ocorrência de todo óbito de mulher durante o período gestacional, parto ou puerpério, e atribui às Secretarias Municipais de Saúde a responsabilidade pela investigação dos óbitos maternos declarados, bem como de todos os óbitos de mulheres em idade fértil Que tenham como causa de morte uma causa presumível de óbito materno ${ }^{(1)}$. Prossegue este mesmo Relatório assinalando Que as ações de reorganização da assistência à gravidez, parto e puerpério, previstas pelo Programa de Humanização do Pré-Natal e Nascimento na região Metropolitana, podem provocar um grande impacto na redução da mortalidade materna no Estado do Rio de Janeiro.

Este programa vem sendo descrito por gestores de saúde, profissionais de saúde e feministas, como um programa estratégico Que pode influenciar na redução das taxas de mortalidade materna. Apesar de ser uma política pública importante, o PHPN não tem sido eficaz para a redução da mortalidade materna, em parte porque não está focalizado na eficácia e segurança dos procedimentos no parto. Além disso, a sua adesão tem sido lenta pelos municípios, segundo dados de 2002.

No Brasil, a morte materna configura-se como um problema de saúde pública. Segundo o Ministério da Saúde ${ }^{(6)}$, as altas taxas de mortalidade materna compõem um Quadro de violação dos direitos humanos de mulheres e de crianças, atingindo desigualmente aquelas das classes sociais com menor ingresso e acesso aos bens sociais, nas várias regiões brasileiras.

O estudo da mortalidade materna tem desafios que superam análises individuais de cada caso e remetem a uma dimensão maior Que é a população e suas Questões de vida, saúde e doença, em uma sociedade onde não existe consenso sobre a igualdade de gênero e direitos reprodutivos ${ }^{(7)}$. Ainda segundo esta mesma fonte, a cada minuto morre no mundo uma mulher em virtude de complicações da gravidez e do parto. Ao fim de um ano, todas essas mortes somam 529 mil, a maioria das Quais ocorre em países em desenvolvimento e poderia ser evitada. Para cada mulher que morre, outras trinta sofrem seqüelas ou problemas crônicos de saúde ${ }^{(7)}$.

Para a implantação do Programa de forma efetiva é necessário contar com o apoio político local e, infelizmente, muitas vezes, esta sanção ainda não é suficientemente coercitiva para levar à obrigatoriedade e prioridade da implantação do $\operatorname{PHPN}^{(7)}$.

Entretanto, apesar da morosidade e da falta de vontade política em alguns municípios, pode-se dizer Que a ação do Ministério Público estadual tem sido positiva, pois acarretou discussão sobre a necessidade de reorganização dos serviços materno-infantis naQuelas localidades, através da assinatura dos Termos de Adesão(2).

Deste processo de implantação do PHPN, surgiu a necessidade de elaboração de planos regionais para mapear os principais problemas nos serviços, levando a uma discussão com gestores e profissionais de saúde sobre a necessidade de melhora da Qualidade dos serviços e a um maior controle de avaliação da Qualidade da assistência durante o pré-natal, parto, puerpério e na assistência de mulheres em situação de abortamento ${ }^{(7)}$.

Mais recentemente, algumas organizações não governamentais internacionais, como o Center for Reproductive Rights e o Cladem (Comitê Latino Americano e do Caribe pela Defesa dos Direito da Mulher) vêm tratando do tema com enfoque nos direitos humanos das mulheres.

Há também alguns relevantes e inovadores estudos acadêmicos Que têm se dedicado a aprofundar a análise da mortalidade materna na perspectiva dos direitos humanos, como os da feminista e professora Rebecca Cook, da Faculdade de Direito da Universidade de Toronto (Canadá) ${ }^{(3)}$.

\section{Morte Materna Evitável X Comitê de Mortalidade Materna}

O tema da mortalidade materna vem sendo tradicionalmente abordado pelo enfoque da saúde pública e coletiva, Que prioriza a investigação epidemiológica das causas de morte materna e os indicadores de Qualidade da atenção à saúde na assistência prestada às mulheres durante o seu ciclo gravídico-puerperal. Por sua vez, os organismos internacionais de supervisão e monitoramento dos tratados internacionais de direitos humanos vêm gradativamente produzindo relatórios Que fazem menção específica à temática da mortalidade materna como violações dos direitos humanos das mulheres $^{(4)}$.

No Brasil, a perspectiva de direitos humanos para o tema da mortalidade materna vem sendo adotada, a partir de uma abordagem política, por organizações feministas de saúde, como a Rede Nacional Feminista de Saúde, Direitos Sexuais e Direitos Reprodutivos, entre outras. A Rede tem produzido documentos e dossiês que são importantes fontes de dados sobre a Questão. Nesse sentido, os movimentos feministas e de mulheres vêm tratando a Questão da mortalidade materna como prioritária para a autonomia e o bem-estar femininos na luta pela sua autodeterminação sexual e reprodutiva $^{(4)}$.

Para estas entidades, a redução das taxas de mortalidade materna no Brasil só será alcançada se os direitos humanos forem garantidos por meio de leis, políticas públicas, mecanismos jurídicos e institucionais eficazes (direitos humanos como um fim). Portanto, a adoção desta perspectiva leva a Que o Estado tome medidas concretas, em especial por meio da implementação de políticas estruturais - Que contemplem ao mesmo tempo a universalidade, especificidade e diversidade das mulheres como sujeitos de direitos humanos - com o propósito de melhorar as condições de vida das mulheres Que são determinantes para aumentar ou diminuir o seu risco de morrer de morte materna evitável (direitos humanos como um meio) $)^{(4)}$.

Nos últimos anos, os esforços governamentais para a redução da mortalidade materna têm sido, ainda, insuficientes. No Brasil, dependendo da região do país, as mulheres não morrem somente pela falta de acesso aos serviços de planejamento familiar, mas também pela falta de Qualidade na assistência prestada durante o ciclo gravídico-puerperal e no abortamento incompleto. Esta má assistência - de baixa Qualidade - inclui o despreparo de uma grande parte de profissionais de saúde no pré-natal, no abortamento, nos momentos críticos de emergência obstétrica e, ainda, a ausência de condições estruturais básicas, como a falta de leitos, equipamentos e transporte para uma unidade com mais recursos ${ }^{(3)}$.

A mortalidade materna no Brasil aponta para a omissão e a falta de diligência do Estado, uma vez Que a maior parte das mortes maternas ocorre por causas evitáveis. A omissão pode ser detectada 
pela falta de implementação de leis e políticas, apontando também para a falta de Qualidade na assistência e para a violência institucional pulverizada na prática de diversos profissionais de saúde. Em geral, esta violência permanece invisível nos serviços, assim como as mortes maternas, como parte da cultura institucional de desvalorização da vida e da saúde das mulheres, Que em sua maioria são de baixa renda, sem escolaridade, mestiças e afrodescendentes. A omissão gera má assistência, discriminação, violência e morte materna evitável ${ }^{(3)}$.

Conforme descrição do Manual dos Comitês de Mortalidade Materna do Ministério da Saúde, destacaram-se causas consideradas relevantes na perspectiva dos direitos humanos ${ }^{(6)}$ :

"Causas profissionais", casos em Que, por falta de capacitação ou capacitação imprópria, tenha ocorrido negligência, imperícia ou imprudência por parte dos profissionais de saúde que atenderam a mulher, como, por exemplo, a não identificação de risco reprodutivo, falha diagnóstica e inadeQuação de procedimentos e tratamentos, entre outros.

"Causas institucionais", casos em Que problemas políticoadministrativos contribuíram para o óbito, tais como falta de captação precoce e ativa da mulher, carência de leitos obstétricos, falta de sangue, de hemoderivados ou medicamentos e inexistência de sistema de referência e contra-referência formalizado para tratamento clínico-ginecológico e obstétrico, entre outros ${ }^{(3)}$.

De acordo com esta mesma fonte, o Estado tem falhado em garantir o pleno funcionamento dos Comitês de Mortalidade Materna, como mecanismo institucional de garantia da política de prevenção das mortes maternas evitáveis e proteção dos direitos humanos das mulheres. Os referidos Comitês podem desempenhar atividades educacionais de promoção e prevenção por meio de sensibilização dos profissionais de saúde e do encaminhamento de denúncias ao Ministério Público para apuração de responsabilidades institucionais nos casos de morte materna evitável(9).

Um outra solução está na análise dos casos de morbidade near miss, aQueles em Que mulheres apresentam complicações potencialmente fatais durante a gravidez, o parto ou o puerpério, mas sobrevivem devido ao acaso ou ao cuidado hospitalar adequado $^{(10)}$. Esta caracterização permite o monitoramento do processo de atenção no atendimento obstétrico e a avaliação da incidência de situações clínicas de risco, podendo romper o processo antes da ocorrência do óbito(II).

Existe ainda um outro problema, Que está relacionado com a identificação dos prontuários clínicos em Que ocorrem estes casos, já Que o profissional não redige o prontuário de maneira adęuada, dificultando esta identificação. É necessário então nas Unidades de Saúde a auditoria aos prontuários, para Que estes profissionais possam tomar conhecimento destes prontuários, e melhorar a sua escrita nos mesmos ${ }^{(12)}$.

O sistema de informações Hospitalares do Sistema Único de Saúde (DATASUS) foi desenvolvido com a finalidade de avaliar o desempenho das unidades, não só com relação às taxas de internação e permanência, mas também de monitorar os índices de mortalidade destas unidades. Esta é uma importante ferramenta para identificação dos casos de mortalidade materna ${ }^{(13)}$. Os gestores Estaduais têm feito esforços no sentido de garantir à Mulher o seu direito à saúde, porém estes têm sido ainda insuficientes ${ }^{(14)}$.

\section{CONSIDERAÇÕES FINAIS}

Apesar do fato de mulheres morrerem em conseQüência da gravidez, parto e puerpério estar entre nós desde que há a presença do homem no planeta, redundantemente continua alvo de muitas discussões, projetos e promessas de cunho político-ideológico. Os governantes conseguem acesso e manutenção no poder público, discursando sobre as necessidades da população como se fossem resolvê-las de modo efetivo, dando aos eleitores falsas idéias de possíveis realizações daQuilo Que eles esperam e precisam.

Nesse sentido, em pleno início do século XXI, ainda se observa a falta de efetividade das políticas propostas, visto Que as mesmas existem, mas não existe nenhum modo de cobrança, e ou avaliação dos profissionais da ponta, os Quais fazem ou deveriam fazer esta política acontecer.

A Enfermagem, edificada e ratificada em objetivo, ética e funções por Florence Nightingale a partir da metade do século XIX, conservou suas bases até os dias de hoje. A notável evolução tecnológica na área biomédica motivou e permitiu à Enfermagem um acompanhamento baseado nos esforços dos seus mestres e profissionais ao longo das contínuas mudanças, Que a cada dia exigem aQuisição mais aprofundada de novos conhecimentos e também contínuas atualizações.

Dentre os dogmas da Enfermagem, podem-se destacar a visão holística da clientela, a visão preventiva, a não contaminação e a separação entre tecnologia e o ser humano em situação de dependente na situação de doença. Em meio à tecnologia do cuidar há a distinção entre números e nomes, resultado da individualização do cliente no processo global do seu atendimento.

Promover e exercer ações preventivas são partes dos aspectos Que caracterizam o ser enfermeiro como cuidador de indivíduos, famílias e comunidades, na saúde e na doença. Esse seu cuidar envolve funções básicas como educativa, administrativa, assistencial, de pesquisa entre outras. A prevenção de morte materna evitável é objetivo crucial nessa abordagem sobre os desafios do enfermeiro numa realidade tão Questionável em termos de atenção à saúde da mulher cidadã de direitos.

A participação da Enfermagem no âmbito da prevenção da mortalidade materna foi reconhecida nas portarias do Ministério da Saúde Que tratam do tema, como fator importante de mudança. Os esforços dos Gestores no sentido de garantir a mulher o seu direito à saúde são evidentes, porém ainda suficientes.

Faz-se necessário então determinar um prazo para Que estas modificações ocorram, pois não podemos, nem devemos mais tolerar mortes maternas evitáveis.

\section{REFERÊNCIAS}

I. Secretaria de Estado de Saúde (RJ). Rede de Centrais de regulação do Estado do Rio de Janeiro. Regulação das ações de saúde: uma idéia em construção. Rio de Janeiro: RJ/ SES/CISA/
NSRCER. Disponível em: http://www.saude.rj.gov.br/ centraisregulacao 
2. Secretaria de Estado da Saúde (RI). Relatório do Comitê Estadual de Prevenção e Controle da Morte Materna e Perinatal. Rio de laneiro: PAISMCA/SES/SS/Superintendência de Saúde Coletiva; 2003.

3. Advocacy. Advocacia cidadã pelos direitos humanos. Rio de Janeiro: Advocacy; 2005.

4. Galli MB, organizador. Mortalidade materna e direitos humanos. Rio de Janeiro: Advocacy; 2005.

5. Lopes GT, organizador. Manual para elaboração de trabalhos acadêmicos. Normas da ABNT - Estilo Vancouver - Bioética. Rio de Janeiro: UERJ/EPUB; 2006.

6. Ministério da Saúde (BR). Programa de Humanização no PréNatal e Nascimento - PHPN.Brasília: SPS/MS, 2006.

7. Palmeira ILT, Lopes MVO. Fenômenos de enfermagem em mulheres atendidas em Serviço de Ginecologia. Rev Enferm UERI 2006; 14(4): 566-73.

8. Martins AL. Mortalidade materna de mulheres negras no Brasil. Cad Saúde Pública 2006; 22(1 I).
9. Schirmer I. A importância da enfermeira nas ações governamentais de redução de morbi mortalidade materna e neonatal. Acta Paul Enferm 2007; 20(3): 5.

10. Pattison RC, Hall M. Near misses: a useful adjunct to maternal death enquires. Br Med Bull 2003; 67(I): 23 I - 43.

II. Ministério da Saúde (BR). Urgências e emergências maternas: guia para diagnóstico e conduta em situações de risco de morte materna. Brasília: Ministério de Saúde; 2000.

12. Amaral E, Luz AG, Souza JPD. A morbidade materna grave na Qualificação da assistência: utopia ou necessidade? Rev Bras Ginecol Obst 2007; 29(9): 484-9.

13. Gomes FA, Mamede MV, Costa JML, Nakano AMS. Morte materna mascarada: um caminho para sua identificação. Acta Paul Enferm 2006; 19(4): 387-93.

14. Sousa MH, Cecatti JG, Hardy EE, Serruya SI. Morte materna declarada e o relacionamento de sistemas de informação em saúde. Rev Saúde Pública 2007; 4 I (2): I8I-9. 